\title{
COMPUTER AIDED DESIGN OF HYBRID CIRCUITS
}

\author{
G.P. FERRARIS, T. CAGNIN, F. FRANCESE, and L. PIANA \\ Advanced Technologies Service, C.A.D., GTE Telecommunicazioni, Cassina \\ de Pecchi, Milano, Italy
}

(Received June 5, 1981; in final form June 26, 1982)

\begin{abstract}
A system (hardware/software) has been implemented which makes it possible, taking advantage of existing programs with the addition of suitable interfaces and appropriate software modules, to automate the design of thick film hybrid circuits.

This system, named FLASH (Fast Layout Approach System for Hybrids), tends to optimize circuit production yields, by basing the design on their degree of complexity in relation with the printing/ firing/trimming cycle.
\end{abstract}

\section{INTRODUCTION}

A system designated Fast Layout Approach System for Hybrids (FLASH) has been developed with the goal of automating thick film hybrid circuit design.

We aimed at creating an interactive tool by which to arrive, starting from the circuit diagram, at the generation of documentation and production tooling, confident that all standards and restrictions demanded by manufacturing processes are adhered to. We wanted also to achieve a satisfactory degree of optimization from both the technical and economic points of view.

A further objective was to attain the greatest possible simplicity in using the system, permitting access to the design engineer without the need for the intermediation of software specialists.

Three different stages can be distinguished in the F.L.A.S.H. system:

1) Resistor design and substrate selection (Figure 1 left)

2) Circuit layout (Figure 1 center)

3) Industrialization (Figure 1 right)

Each stage takes advantage of hardware and software already existing in our company. Stage 1 uses a VAX 11/780 computer (Figure 2 bottom), but it is capable of utilizing any "general purpose" computer. Stage 2 utilizes the SCI-CARDS package for layout of printed circuits, implemented on VAX 11/780. Stage 3 uses the interactive CALMA graphic system (Figure 2 top) for the capture, process and management of graphic files.

Some software modules have been integrally developed. For the remaining part we have taken advantage of existing software, developing suitable interfaces towards the FLASH. We aimed at the development of a system having the following basic characteristics:

- Interactive: to facilitate its use for circuit designers and to ensure the utmost flexibility.

- Modular: to facilitate the possibility of expansion

- Parametric: to easily adapt it to developments in the technological processes of hybrid circuit manufacture.

We ended up with a custom-built and extremely powerful system in the layout and industrialization stages. 


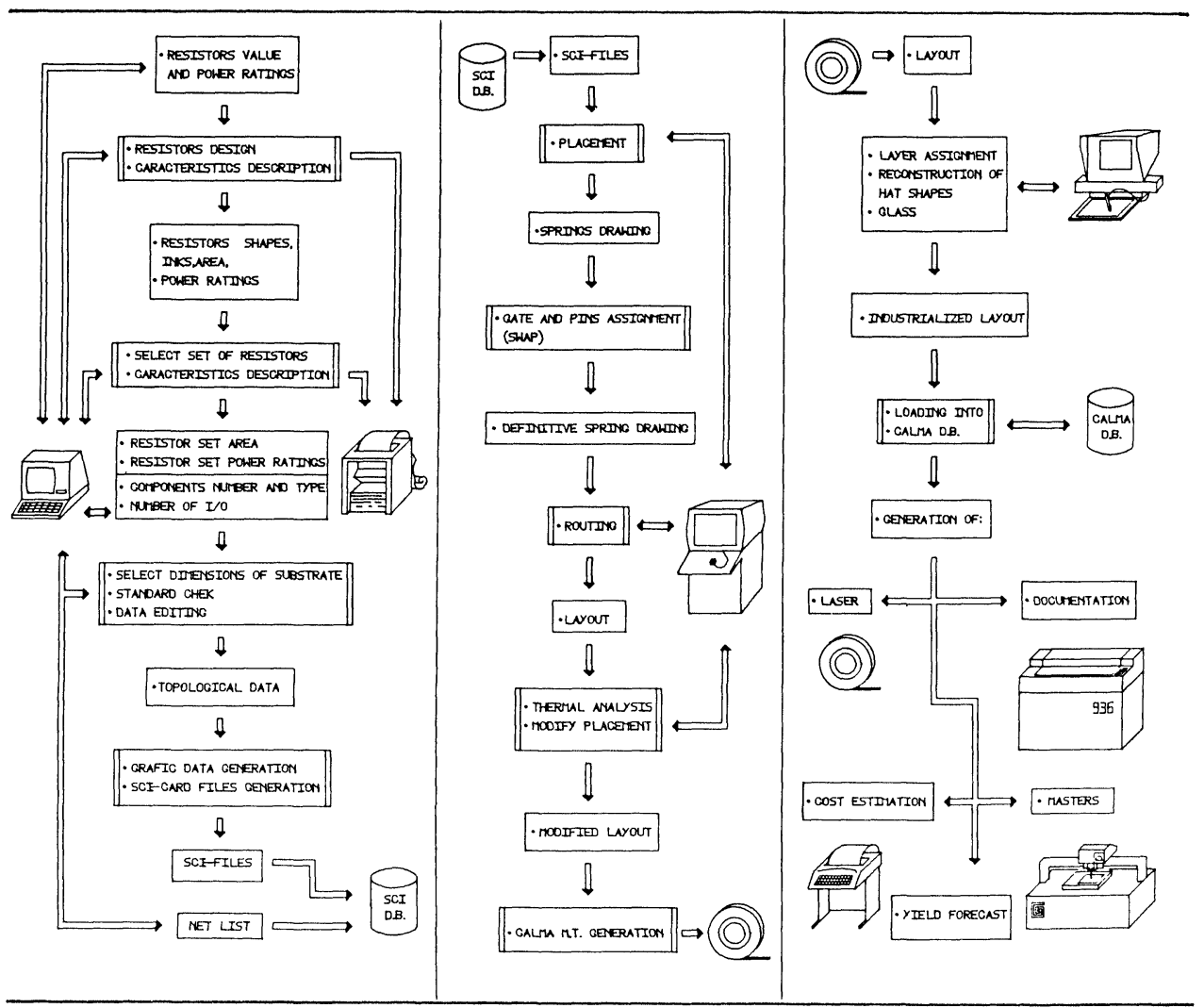

FIGURE 1 The F.L.A.S.H. system.

\section{RESISTOR DESIGN AND SUBSTRATE SELECTION}

It is a well known fact that there are process factors (screen printing, furnace temperature and stability and trimming methods and procedures), factors connected with the materials (ink types, termination effects, temperature coefficient of resistors, etc.) as well as factors relating to the suppliers (product guarantee, reproducibility of the batches, which influence the proportioning of thick film resistors. All these factors have been weighed, in setting up the technology, both qualitatively and quantitatively, on a mathematic and statistical basis. The obtained numerical values have been integrated in a series of interactive programs written in FORTRAN IV language, and they represent a guide to the design of thick film resistors. Their main task is therefore to illustrate, to the designer, all the available possibilities and at the same time to verify that his choices comply with accepted standards.

Starting from ohmic values and resistor power ratings the system verifies that the designer respects the standard limits of length, width, power density and number of squares allowing him, however, the possibility of derogating from these restrictions in special cases.

Hence it goes on to calculate all the possible shape-solutions, with all the different inks available and the relative area taken up for each resistive value, taking into account correction factors due to termination effects (Figure 3). ${ }^{1}$ 

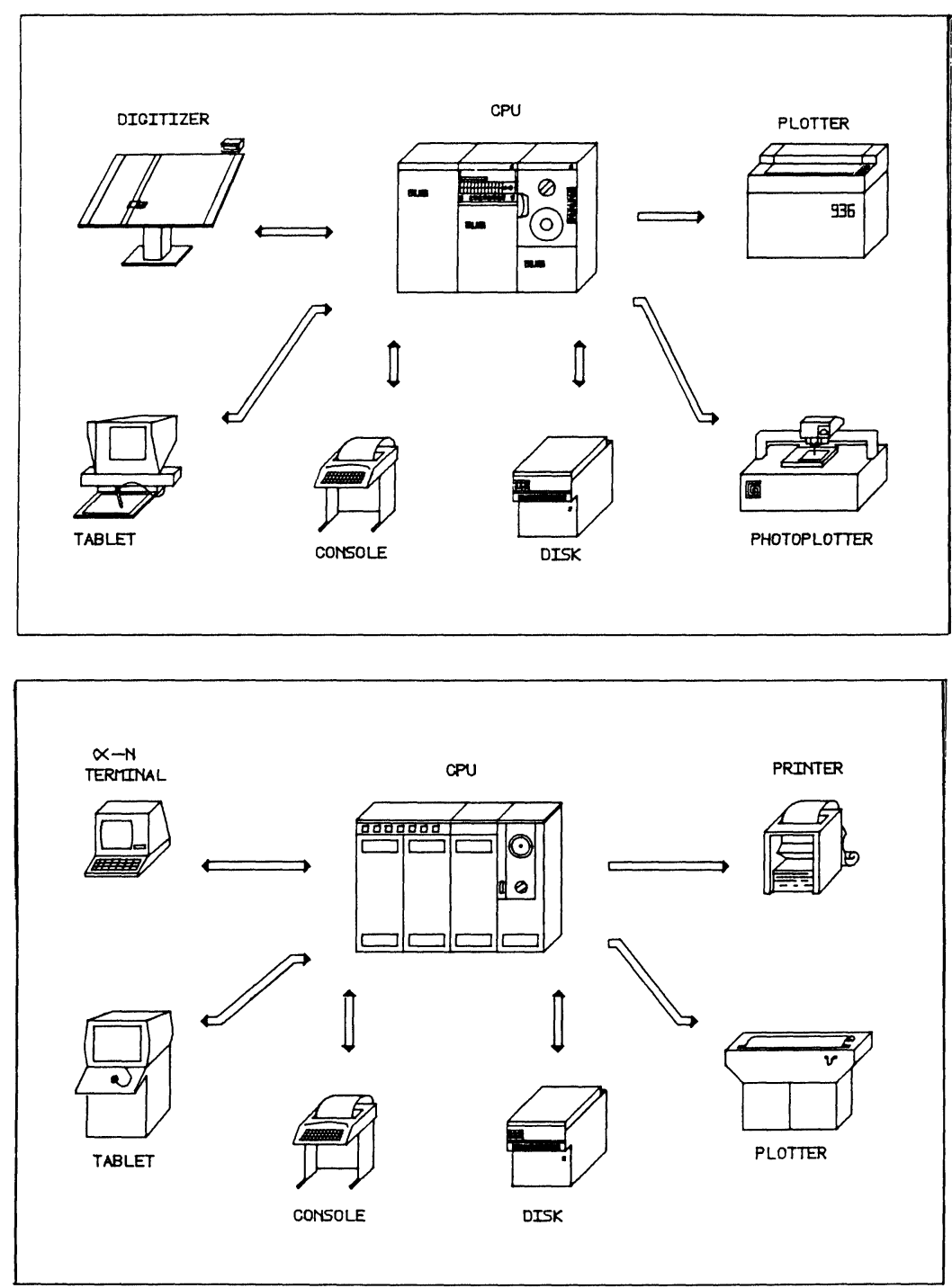

FIGURE 2 The CALMA graphic system (top); the SCI-CARDS system (bottom).

Those resistors, whose dimensions would result in being below the minimum dimensional limits, are automatically increased by the system (with a consequent increase in power rating) until they fit the standard limits.

The system then proposes a series of resistors. It chooses them from among those possible by using an algorithm which quantifies the problems connected with the manufacturing processes by means of a parameter defined as Difficulty Degree (DD), described in the appendix. Should the proposed solution be unacceptable to the designer, he can choose a different one from among all those possible.

In this situation the system describes the solutions to him one-by-one and therefore provides him, for the one chosen, with all the information concerning resistor drift (Figure 4), TCR, ${ }^{2}$ noise factor, etc. 
LOU. NUMBEK, $552-900 / 14$

RESIEIIUK N., 4, VAL.UL (KOIHM), I 10.001

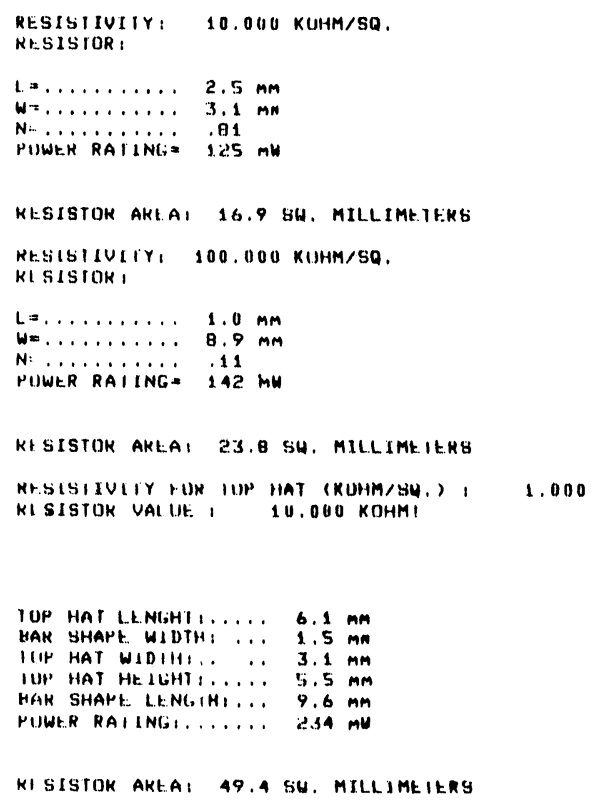

FIGURE 3 Calculated shape-solutions for a 10. Kohm resistor.

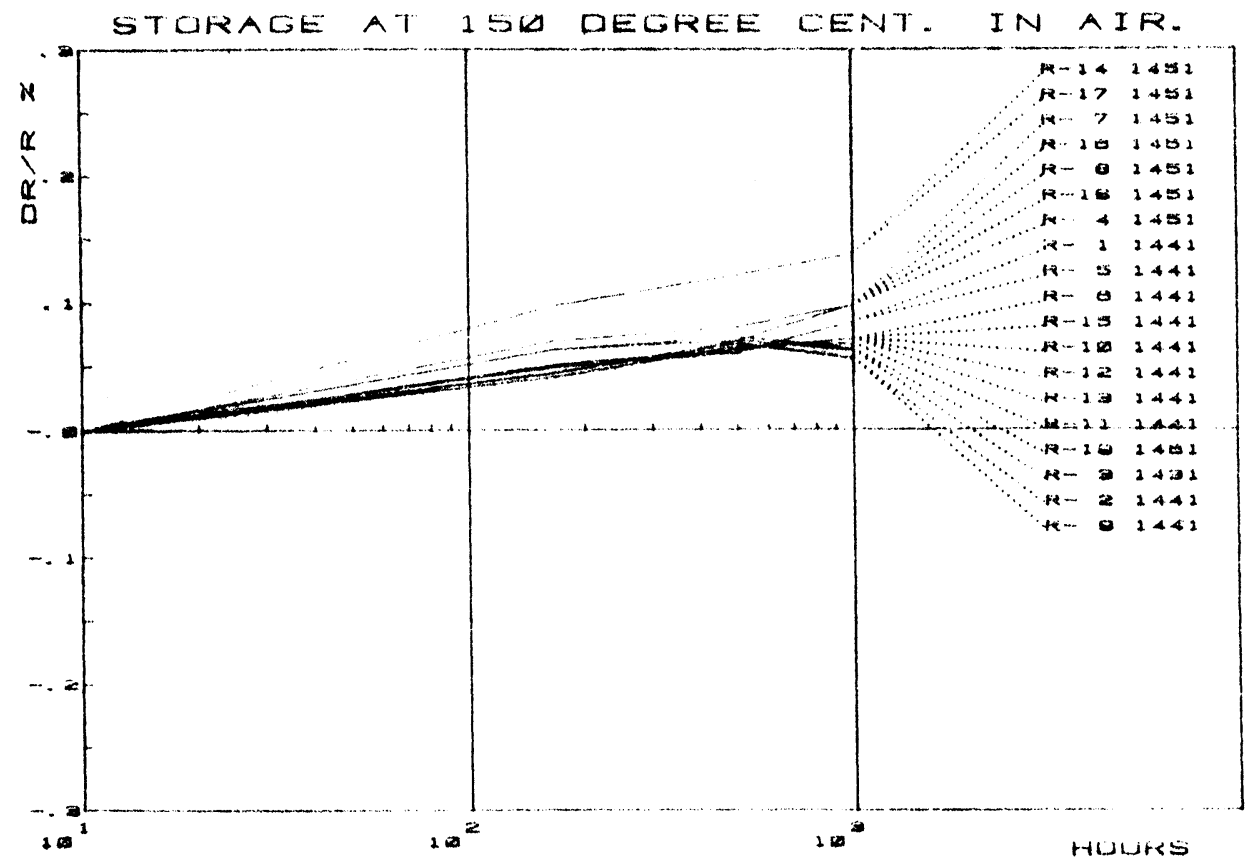

FIGURE 4 Resistors drift for the selected series of Frequency Generator. 
At this point the system, taking into account the overall substrate area taken up by resistors, mounted components, input/output (I/O) areas and the estimated connection area requirements, suggests the smallest usable substrate from the standard sizes and power handling capabilities. ${ }^{3}$ Even in this case the designer can modify the choice.

At the end of this stage an interface program proceeds automatically to the production of the input data for the next stage which depends on the SCI-CARDS package.

\section{CIRCUIT LAYOUT}

The SCI-CARDS package was conceived for printed circuit layout design. Thick film hybrid circuit, except some limitations, can be treated as multilayer printed circuits. The input data to this package are of two types: library-files and user-files.

The library-files data define the physical and electrical characteristics of the components. For printed circuits they are constant (the physical dimensions of a resistor do not vary with its ohmic value if power rating remains unchanged).

Thick film resistors physical characteristics, on the other hand, are variable. Therefore we had to write programs which create and manage these libraries automatically so as to make them visible to the user. These programs also store them on magnetic tape, once design has been completed, in order to optimize memory occupancy.

The user files, instead, contain information concerning the substrate size, number of $\mathrm{I} / \mathrm{O}$ areas, number and types of components, net list and path dimensions. These files too have to be created automatically at the end of the design stage so that the user sees only the interactive stage of the SCI-CARDS package relative to circuit problems and not the one relative to software problems.

When all the input data are available to the SCI-CARDS package, it is extremely interactive and design oriented. All the instructions are encoded on specially preformed menus and all the designer has to do is to point with the tablet cursor to the operation he intends to perform. These operations may be grouped into three headings:

- Component placement

- Swap

- Routing

Component placement can be completed automatically or interactively.

In the latter case considerable assistance may be afforded by the fact that the substrate outline, I/O areas, component outlines and point-to-point connections to be made (springs), can be visualized on the cathode ray tube (CRT) screen (Figure 5).

An algorithm can also be used which optimizes placement in order to avoid, or at least to minimize, cross-overs. ${ }^{4}$

The swap phase is particularly important for circuits which mount multifunction IC's. In fact the system cyclically tries all the possible pin, gate and mechanical package permutations in order to achieve, by successive approximations, the minimum connection lengths.

Finally the routing program makes all the connections among the various components while respecting all required spacings and other specified restrictions.

For this stage we depended heavily on home made software due to the fact that the SCI-CARDS being oriented for the layout design of printed circuits, does not envisage thick film restrictions and typically the passing of conductors beneath resistors, top-hat geometries, etc. However, there are different ways of achieving a routing on the SCICARDS level. Best results for low density circuits were obtained by running the paths on 


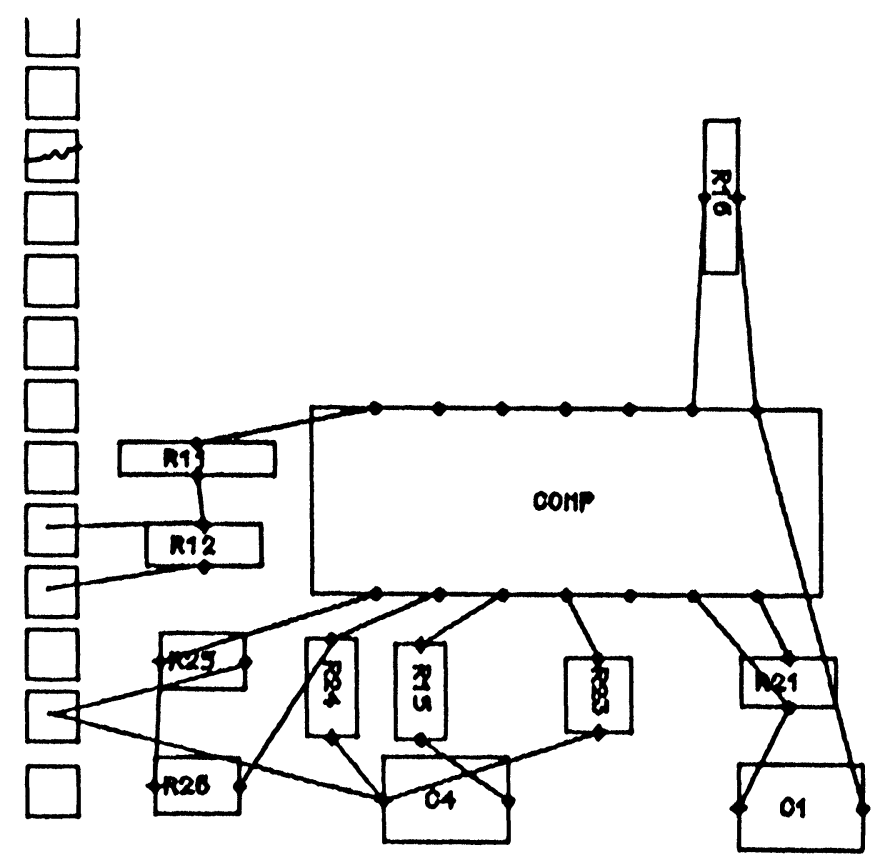

FIGURE 5 Point to point component connections visualized on CRT by "springs".

a single layer and performing an eventual completion interactively. Interactive editing proves anyway extremely powerful, since it is impossible to make connection mistakes (the system has a list of all the connections) and all spacings and other demands are always respected. Moreover by operating on a refresh cathode ray tube, all graphic operations described above are visualized in real time. For high density circuits, on the other hand, it proved more convenient to make the routing directly on two layers, thus simulating cross-overs. Once the layout has been accomplished an interface program generates the magnetic tape for the next stage which takes place on a CALMA system.

\section{INDUSTRIALIZATION}

The CALMA SYSTEM was designed for the capture and the management of graphic files. These files can be easily manipulated by using the tablet or the digitizer and the feedback of all operations can be evaluated immediately on a cathode ray tube.

One of the main features of the CALMA system is its ability to handle any kind of structure with data located on one or more of its 29 layers.

Therefore it proves particularly convenient where multilayer circuits of any kind are involved.

As far as our case is concerned, the passing from SCI Data base to CALMA Data base does not represent simply a format transformation, but it consists in making it adopt the thick film philosophy, viz. different layers for different inks, replacement of rectangular geometries with top-hat geometries, overglaze etc. 
These operations are performed in an interactive manner through a series of programs in GPL language (analogous to APL) which in part take advantage of the system features and in part take advantage of the information coming from the design stage.

Once transfer has been completed, the CALMA data base contains all the topological information pertaining to the circuit. Therefore it is possible to get photographic reproductions of the layouts in a 1:1 scale directly (masters) which are used to generate the screen-printing frames, paper documentation for the manufacturing processes and archives, instruction manuals, instructions for laser resistor trimming, statistical data relating to the design and manufacture as well as data required for cost computation.

\section{EXAMPLE AND CONCLUSIONS}

The FLASH system has been applied in the design of a module of considerable density (Frequency Generator) whose electrical diagram is shown in Figure 6.

It was implemented on two layers with 12 cross-overs (Figure 7).

Design automation allows a strict observance of those standards that ensure a product of superior quality, high production yield and fewer modifications.

Moreover a tangible amount of labour saving and reduction in the elapsed time between the completion of the electrical design and the preparation of the prototypes, is achieved during the design stages.

With the experience gained from the module in the above example as well as from other modules we deduced that a saving of some $80 \%$ in design costs was achieved and the number of trials passes was reduced from 2.6 to 2 .

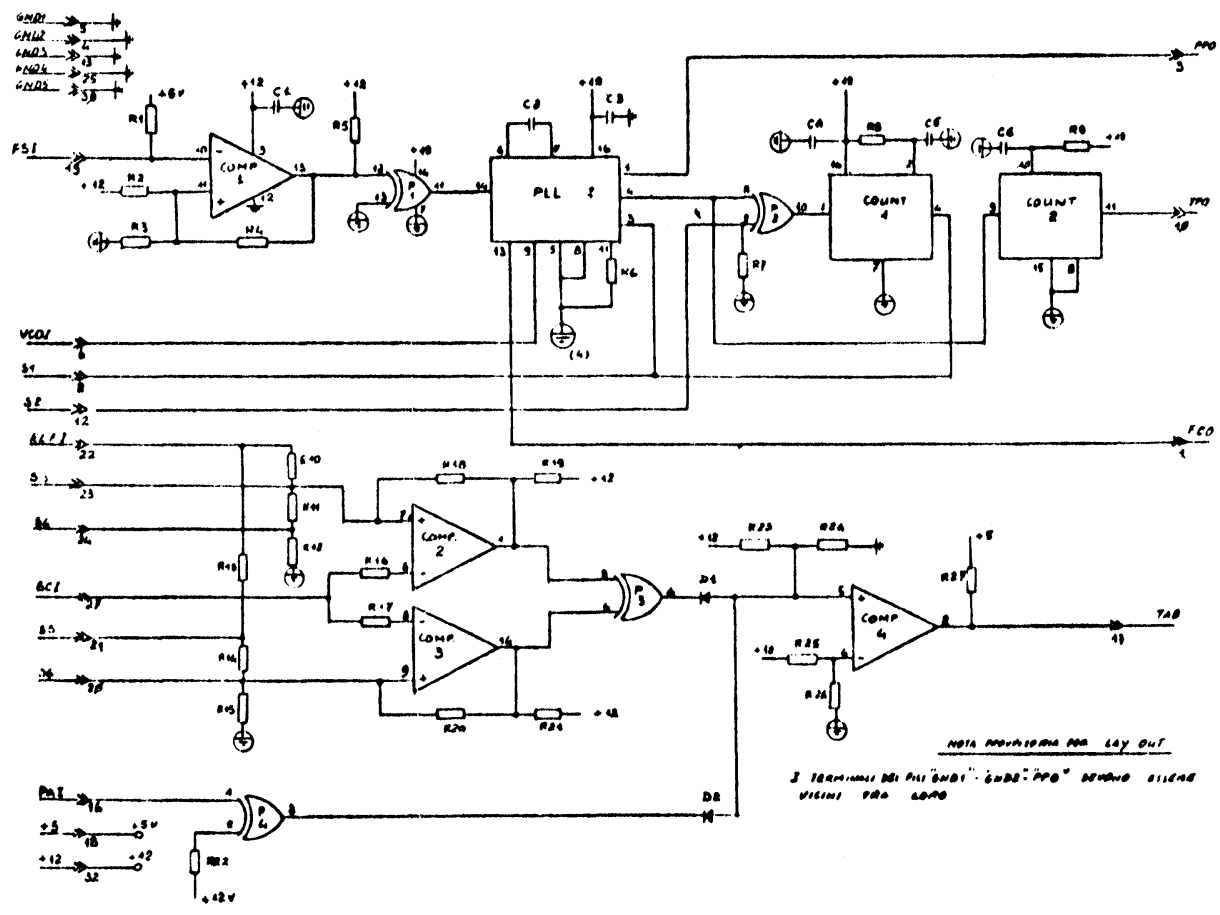

FIGURE 6 Frequency Generator Module (electrical diagram). 


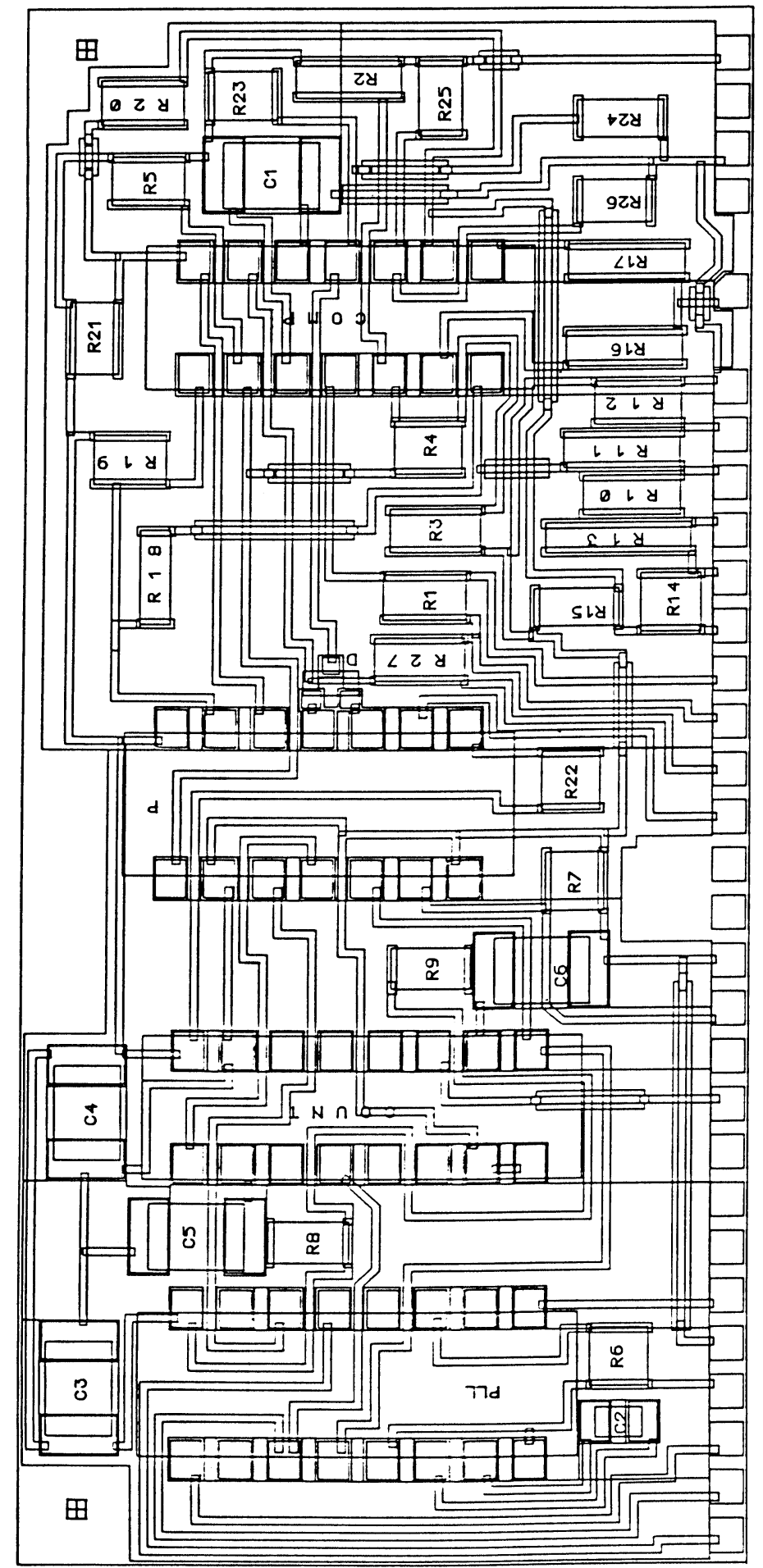

FIGURE 7 Layout of Frequency Generator Module. Size $82.55 \times 38.1 \mathrm{~mm}^{2}$. 


\section{APPENDIX 'DIFFICULTY DEGREE’ CLASSIFICATION}

We have seen in the previous paragraph that one of the things made possible by the programs is to select an optimal set of resistive layers capable of satisfying the implementation of all resistors in the circuit concerned. It was therefore necessary to provide the computer with the selection criteria.

A method for classifying thick film in terms of the complexity in the single hybrid circuit has been developed in an effort to subdivide both new and existing circuits into easy and difficult categories from a manufacturing point of view. ${ }^{5}$ For the print/fire/trim cycle, as an example, the rating method used consists in reviewing each print layer individually and assigning a rating of between one and three to each print. The circuit's Difficulty Degree is the sum of the points for each layer of artwork and indicates the probability of manufacturing errors which would result in thick film scrap, so that it is possible to plot yield versus Difficulty Degree in a curve like the one represented in Figure 8.

Obviously the rating criterion is somewhat arbitrary and obviously it is strongly connected with the status of the manufacturing facility.

Moreover it can be modified by feed-back of information from the manufacturing facility itself to obtain a better correlation between actual yield and theoretically calculated Difficulty Degree.

Specifically for the resistive layers the following rating scale was decided on:

resistors - Rated I, II, or III (each resistor layer rated separately).

I - Simple geometries with less than 10 resistors on a given layer.

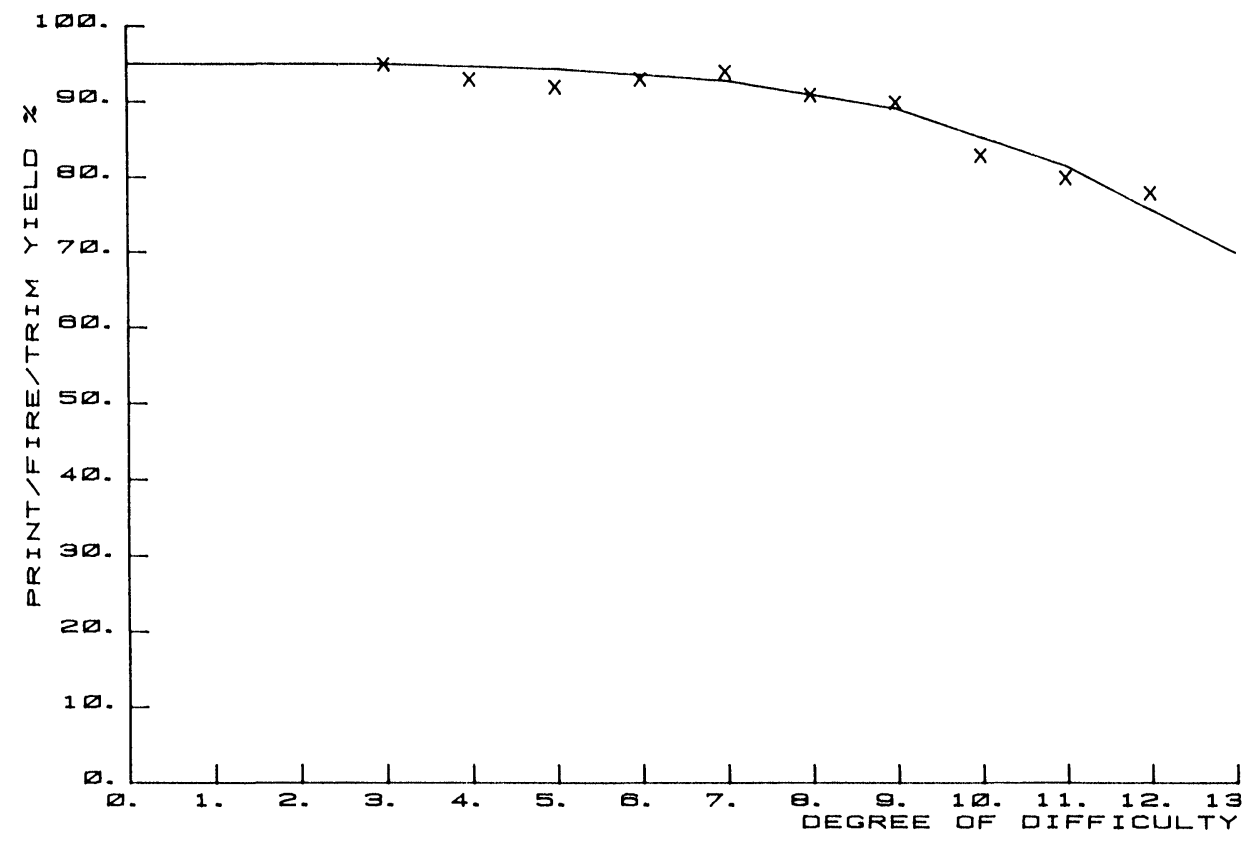

FIGURE 8 Manufacturing yield versus Difficulty Degree. 
II - All $10 \mathrm{ohm}$ or 1 megohm layers.

- All top hats.

- Resistors of very small geometry.

- Two different resistors terminating on the same pad (this should be elimated for new designs).

- Resistors of very large geometry (pinholes).

III - Two of the conditions listed under II.

For example, for the circuit in Figure 7 (Frequency Module) the selection of the resistive layers involves a $\mathrm{DD}=5$. Adding the rate for conductors, cross-over and glaze layers we obtained a total $\mathrm{DD}=11$ with a predicted yield of $87 \%$.

\section{REFERENCES}

1. K.E. Smith and S. Belochi, "Thick film resistor design for hybrid substrates" Proceedings of the Fifth GTE Symposium on CAD/CAM, Mountain View, pp. 335-347 (April 24, 25, 26 1978).

2. G.P. Ferraris, F. Francese, O. Sternfeld, "Film spesso: si o no per circuiti critici?" (Geometries and terminations effect on T.C.R.) (in Italian), Giornate di Studio su: Affidabilita, Applicazioni e Tecniche di Produzione dei Circuiti Integrati Ibridi, Milano (5-6 ottobre 1978).

3. G.P. Ferraris, M. Tudanca, "The solution of two-dimensional heat conduction problems for predicting operating temperature and power handling capabilities of hybrid circuits," Electrocomponent Science and Technology, vol. 7 pp. 97-105 (1980).

4. R. Van Der Leeden, W. Ulbrich, "A new approach to the topological design of hybrid circuits" European Hybrid Microelectronics Conference 1979, Ghent, Belgium, pp. 31-46 (21, 22, 23 May).

5. J. Floegel, "Hybrid-degree of difficulty," GTE Inter Office Correspondence, (April 24, 1978). 

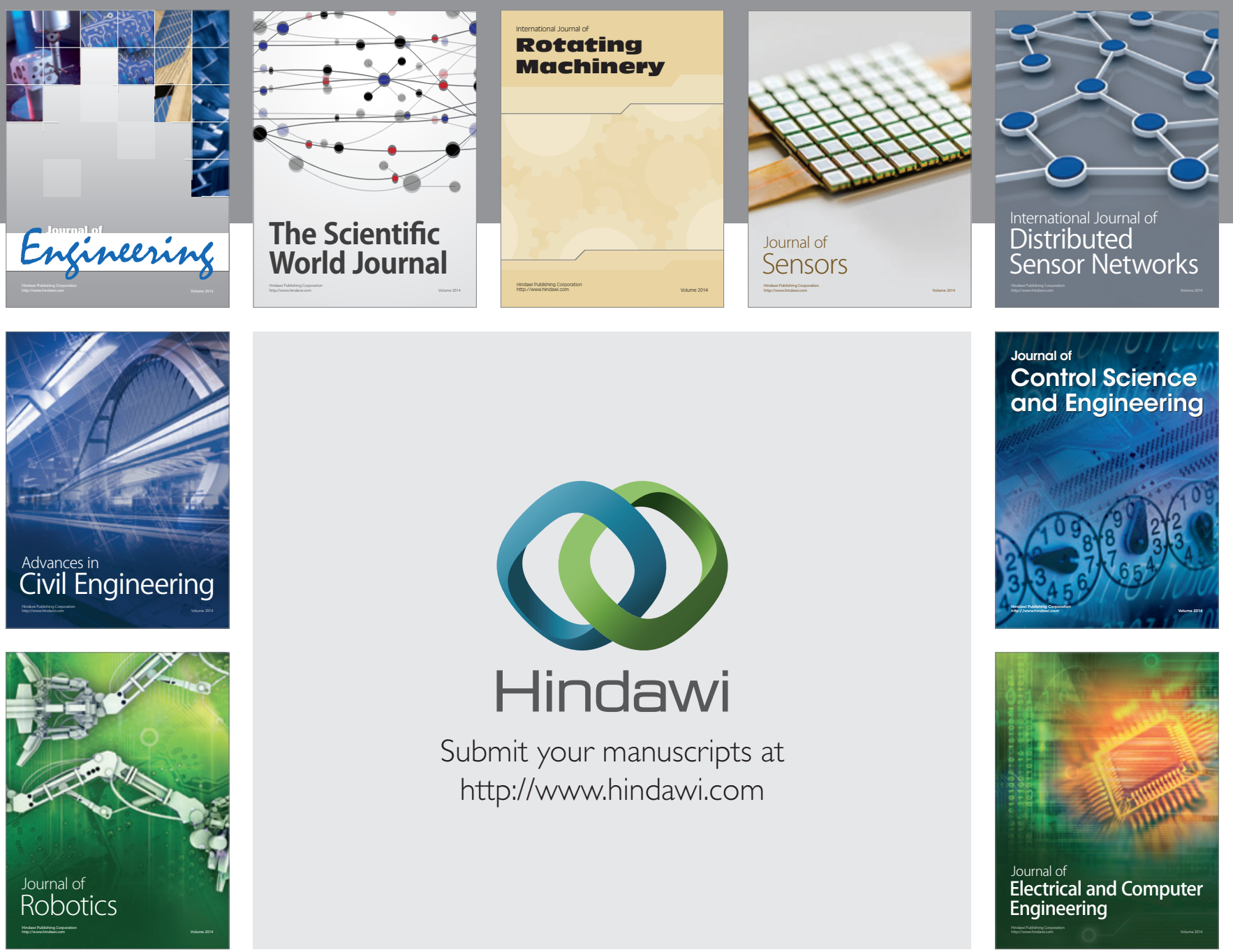

Submit your manuscripts at

http://www.hindawi.com
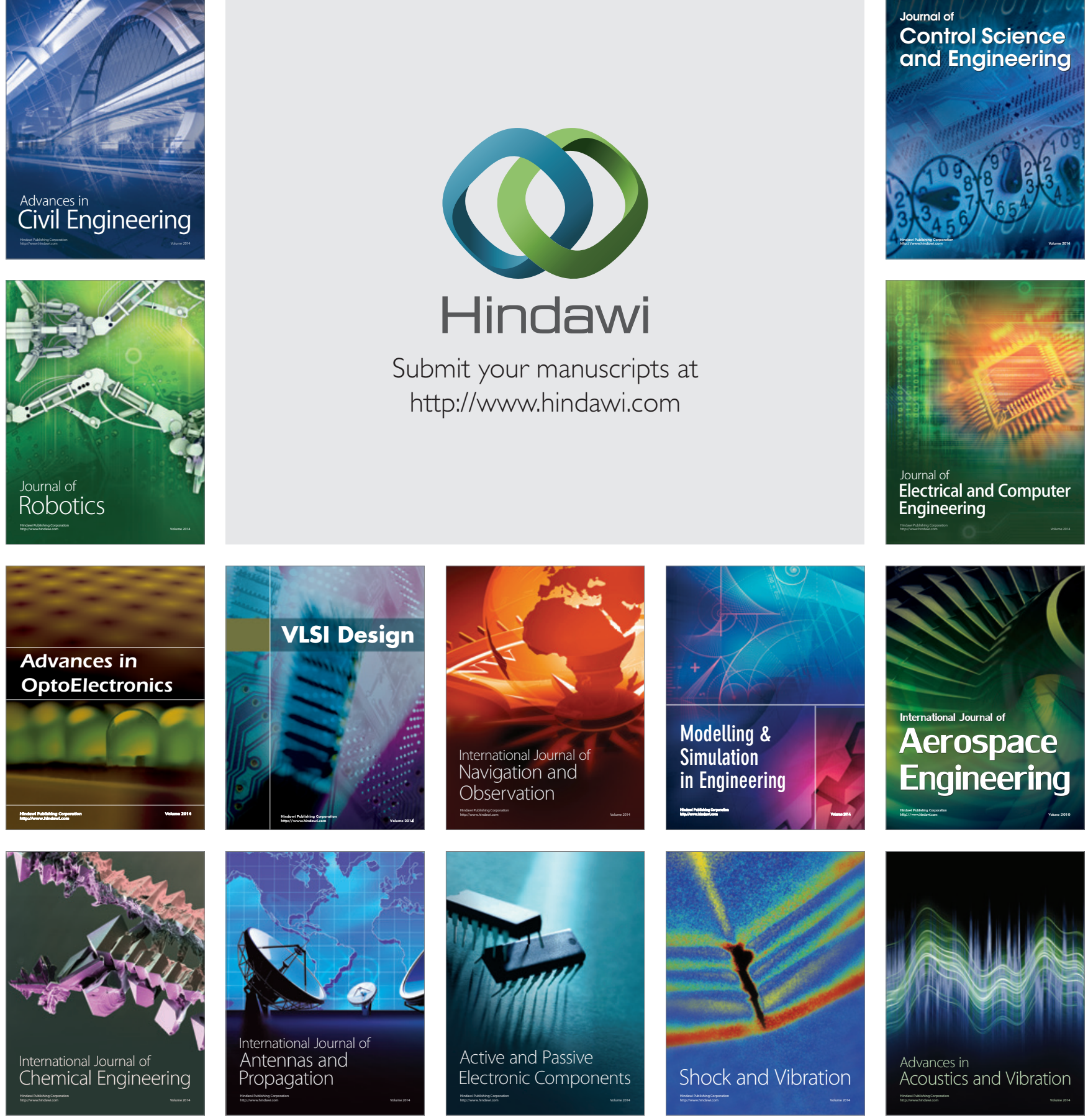